\title{
Austerität in Deutschland und Europa
}

\author{
Sebastian Klauke
}

Online publiziert: 31. Januar 2020

(C) Der/die Autor(en) 2020

Sturm, Roland, Tim Griebel, und Thorsten Winkelmann (eds.) (2017): Austerity: A Journey to an Unknown Territory. Discourses, Economics and Politics. ZfP Zeitschrift für Politik Special Issue 8. Baden-Baden: Nomos. 306 Seiten. 59,00€

Petzold, Tino (2018): Austerity Forever?! Die Normalisierung der Austerität in der BRD. Münster: Westfälisches Dampfboot. 292 Seiten. 30,00€

Das Wort Austerität stammt aus dem Griechischen und bedeutet ursprünglich so viel wie Strenge und Herbheit. In wirtschafts- und gesellschaftspolitischer Hinsicht geht es um (staatliche) Sparpolitik und Haushaltsdisziplin und findet seit Mitte des 20. Jahrhunderts Anwendung. Und diese Bedeutungsdimensionen sind es, die im Zusammenhang der Krise seit $2007 \mathrm{ff}$. den Begriff bzw. das Konzept über rein wirtschafts- und politikwissenschaftliche Kreise hinaus bekannt gemacht haben und auch zu vermehrter wissenschaftlicher Aufmerksamkeit führen.

Die Zeitschrift für Politik widmet dem Thema ein Special Issue in englischer Sprache, das neben einer Einleitung der Herausgeber zwölf sehr verschiedene Beiträge umfasst. In der Einleitung unterbleibt eine prinzipielle Definition von Austerität, es sei eben eine Frage der wissenschaftstheoretischen Perspektive, deren Varianten und Ansatzpunkte kurz aufgeführt werden. Im Grunde soll damit ausgedrückt werden: Es gibt ganz verschiedene Herangehensweisen, das Themenfeld zu erforschen. Austerität wird damit als ein zu untersuchendes Phänomen hingenommen. Als Begründung verweist die Redaktion auf einen „eclectic approach“ (S. 8), um das Zusammenführen der verschiedenen Beiträge in einem Heft zu rechtfertigen. Denn ,eclectic scholarship“ sei ,the best companion for a diversified group of researchers on their way to a better understanding of economic phenomena in general

S. Klauke $(\bowtie)$

Kiel, Deutschland

E-Mail:klauke@ips.uni-kiel.de 
and of austerity in particular“ (S. 9). Genau hier liegt das grundlegende Problem des Bands: Der Begriff der Austerität ist die einzige Klammer, die ihn zusammenhält. Eine Diskussion oder Bezugnahme der Beiträge untereinander, eine gegenseitige Wahrnehmung findet nicht statt, womit eine große Chance vertan wird.

Die schiere Bandbreite der Beiträge entschädigt insofern, als ganz unterschiedliche Methoden, Analysen und Vorgehensweisen präsentiert werden, aus deren Fundus man sich dann bedienen und inspirieren lassen kann. Um einen Eindruck hiervon zu gewähren, seien die folgenden Beiträge genannt:

Bob Jessop nähert sich dem Thema aus der Perspektive der maßgeblich von ihm und Ngai-Ling Sum entwickelten Cultural Political Economy (CPE) und unterscheidet ,austerity policies“, ,politics of austerity“ und ,austerity state“ (S. 29), womit die drei klassischen politikwissenschaftlichen Betrachtungsebenen Polity, Policy und Politics benannt sind. Es folgt ein fokussierter „Ritt durch die Geschichte“ - vom kurzen goldenen Zeitalter des Fordismus zum Neoliberalismus und seinen verschiedenen Ausprägungen hin zur Krise der Jahre $2007 \mathrm{ff}$. sowie den Entwicklungen in Griechenland. Die CPE ist eine Verbindung von Marxismus (Marx, Gramsci, Althusser, Poulantzas), heterodoxen Ansätzen der Ökonomie sowie der foucaultschen Diskursanalyse und fokussiert auf Semisiosis im Sinne von ,sense- und meaningmaking“. Der Artikel stellt einen gelungenen Beitrag dieser Theorierichtung dar.

Tim Griebel bedient sich mit Fokus auf Deutschland ebenfalls der CPE und stellt eine „Corpus-Assisted Critical-Realist Discourse Analysis“ vor. Die Besonderheit seines Beitrags liegt in der Erweiterung der Perspektive um Erich Fromm und eine materialistisch soziale Psychologie, also in einer Verknüpfung von Materialismus und Psychologie (S. 53). Dies soll dem Ziel dienen, besser nachzuvollziehen, weshalb Menschen bestimmten ,imaginaries“ stärker anhängen als anderen (S. 53). Er wertet Artikel der Welt und der taz (1. Januar 2007 bis 31. Dezember 2015) sowie Veröffentlichungen der deutschen Regierung aus und verknüpft dabei Quantitative Corpus Linguistics und Critical Discourse Analysis (S. 58-59).

Reiner Grundmann, Kim-Sue Kreischer und Mike Scott beleuchten den titelgebenden „Discourse of Austerity in the British Press“. Sie legen exakte Rechenschaft über ihr methodologisches Vorgehen ab, das dem Ziel, to construct a valid corpus on the discourse of austerity“ (S. 127) dient. Zentrale Akteure in der britischen Diskussion waren das - hierzulande wohl kaum bekannte - Office for Budget Responsibility und der konservative Politiker George Osbourne.

Vassilis K. Fouskas und Constantine Dimoulas machen in ihrem Beitrag „Imperial Bondage: Austerity in Greece, 2008-2018“ darauf aufmerksam, dass die Austeritätspolitik nicht erst mit der Krise 2007 ff. begann (S. 191), konzentrieren sich dann aber auf die Untersuchung der spezifischen Austerität in Griechenland: eine ,,most peculiar and acute form of bondage, that of internal devaluation", ,a direct colonial regime" unter der direkten Kontrolle durch die Troika (S. 192). Demokratie spielte keine Rolle mehr und Maßnahmen, die die allgemeine wirtschaftliche Lage verbessern sollten, haben bis 2017 keine nennenswerten Erfolge vorzuweisen (S. 211). Was jetzt - nach der Abwahl von Syriza - geschieht, bleibt abzuwarten.

Brian Girvin untersucht die „Political Consequences of Austerity in the Republic of Ireland: Domestic Choice, International Constraints and Democratic Deficits“. Es handelt sich um einen Nachvollzug der Entwicklung, die in der aktuellen Frag- 
mentierung des Parteiensystems endete, mit all den damit verbundenen Problemen der Regierungsbildung sowie für das eigentliche Regieren. Es kam zu einer Stärkung der linken Parteien, die aber stark zersplittert sind und deren Stimmanteil im Untersuchungszeitraum bei 30 Prozent lag.

Was in dem Special Issue nur randständig aufblitzt, macht Tino Petzold in einer auf seiner Dissertation basierenden Darstellung eindringlich deutlich: Austerität ist kein Phänomen der vergangenen zehn bis zwölf Jahre, sondern ist in Deutschland seit Jahrzehnten Kern der staatlichen Politik. Petzold schärft also den Blick für die historische Perspektive. Würde man nur die Entwicklung seit $2007 \mathrm{ff}$. in den Blick nehmen, geriete die tatsächliche Genese der Austeritätspolitik überhaupt nicht in den Blick (S. 83).

Das Buch umfasst sechs Kapitel. Ausgangspunkt ist die Feststellung einer aktuell normalisierten Austerität, worunter der Autor ,die historisch-geographisch spezifische Gestalt des finanzpolitischen Systems strategischer Selektivität, in dem staatliche Haushalsdisziplin privilegiert und auf Dauer gestellt wird“ (S. 14), versteht. In der Analyse, wie es dazu kam, stützt er sich auf den Begriff des Systems der strategischen Selektivität, wie er in der materialistischen Staatstheorie Verwendung findet (S. 14). Weitere Grundlagen sind die materialistische Rechtstheorie sowie die ScaleDebatte (S. 15), d.h. die räumlichen Dimensionen der Geografie des Steuerstaats geraten in den Blick. Diese drei Ebenen sind es, auf denen Petzold die Entwicklung der Austerität mithilfe einer ,historisch-räumlichen Pfadanalyse“ (S. 16) in (West-)Deutschland von Ende der 1940er-Jahre bis in das Jahr 2013 nachvollzieht. Austerität versteht der Autor dabei gleichermaßen als „Idee, als politisches Projekt, als fiskalisches Regime sowie als Geographie“ (S. 17).

In Kapitel 2 definiert Petzold die grundlegenden Dimensionen von Staat, Recht und Raum der Austerität (S. 18-78) und stellt dann im dritten Kapitel die historischmaterialistische Politikanalyse der Normalisierung von Austerität dar (S. 79-95), womit u. a. eine Diskursanalyse des Rechts verbunden ist. Es folgt die empirische Darstellung der politischen und juristischen Prozesse der Normalisierung anhand des Beispiels der Bundesrepublik Deutschland (BRD) von 1949 bis 2004 (S. 96-184). Zentrales Ergebnis ist, dass die Politik der Austerität in Deutschland seit den 1970erJahren immer wiederkehrt (S. 96). Seit Ende des letzten Jahrzehnts wurde die ,dauerhafte Krise der öffentlichen Finanzen“ (S. 181) herbeigeführt. Es waren insbesondere die umfangreichen Steuersenkungen der Regierung Schröder (S. 171), die die Reproduktion des Austeritätsregimes ermöglichten (S. 172). Im fünften Kapitel stehen das Recht und die Entwicklung der Schuldenbremse sowie des europäisches Fiskalpakts im Fokus: Es geht um die Verkettung der verschiedenen räumlichen Ebenen von Deutschland und Europa bzw. der Europäischen Union (EU) in den Jahren 2005 bis 2013, verbunden mit der Beschränkung von Parlamentsrechten sowie der Stärkung ausführender Organe (S. 185-266). Abgeschlossen wird das Buch durch ein kurzes Resümee.

In allen Kapiteln hat der Autor neben den bundesrepublikanischen Entwicklungen immer auch die Prozesse auf der europäischen Ebene im Blick und löst seinen Anspruch ein, die drei einführend genannten Dimensionen in die Analyse miteinzubeziehen. Somit handelt es sich ebenfalls um einen Beitrag zur (kritischen) Europaforschung. Für eine bessere Orientierung und Einordnung wäre ein vergleichender 
Blick auf andere Länder der EU wünschenswert gewesen. Die mitunter schwierigen Sachverhalte und das rechtliche Vokabular wären in einem insgesamt sprachlich eingänglicher und noch besser formulierten Text wirkungsvoller zur Geltung gekommen.

Und auch wenn man der Analyse vor allem in ihren methodischen Grundlagen nicht zu folgen vermag, formuliert die Monografie eine ernstzunehmende Mahnung an die Politikwissenschaft eindringlich: Die Auseinandersetzung mit Recht, Rechtsetzung und Rechtsprechung ist aus politikwissenschaftlicher Perspektive grundlegend notwendig. Darüber hinaus öffnet Petzold den Blick auf die historische Entwicklung der Austeritätspolitik und zeigt auf, wie sehr die aktuelle Lage von teilweise jahrzehntealten politischen wie juristischen Auseinandersetzungen und Entscheidungen geprägt wird. Das von ihm entwickelte und verwendete methodologische Instrumentarium wird hoffentlich in weiteren Analysen Anwendung finden. Die Kopplung und Diskussion mit den Beiträgen des Special Issues wäre wünschenswert.

Funding Open Access funding provided by Projekt DEAL.

Open Access Dieser Artikel wird unter der Creative Commons Namensnennung 4.0 International Lizenz veröffentlicht, welche die Nutzung, Vervielfältigung, Bearbeitung, Verbreitung und Wiedergabe in jeglichem Medium und Format erlaubt, sofern Sie den/die ursprünglichen Autor(en) und die Quelle ordnungsgemäß nennen, einen Link zur Creative Commons Lizenz beifügen und angeben, ob Änderungen vorgenommen wurden.

Die in diesem Artikel enthaltenen Bilder und sonstiges Drittmaterial unterliegen ebenfalls der genannten Creative Commons Lizenz, sofern sich aus der Abbildungslegende nichts anderes ergibt. Sofern das betreffende Material nicht unter der genannten Creative Commons Lizenz steht und die betreffende Handlung nicht nach gesetzlichen Vorschriften erlaubt ist, ist für die oben aufgeführten Weiterverwendungen des Materials die Einwilligung des jeweiligen Rechteinhabers einzuholen.

Weitere Details zur Lizenz entnehmen Sie bitte der Lizenzinformation auf http://creativecommons.org/ licenses/by/4.0/deed.de. 